\title{
Cuantificación del contenido de compuestos bioactivos y actividad antioxidante in- vitro en extractos de flor de Jamaica (Hibiscus sabdariffa)
}

\section{Quantification of the content of bioactive compounds and in-vitro antioxidant activity in extracts of Jamaica flower (Hibiscus sabdariffa)}

\author{
Erick N. Valdez-López ${ }^{a}$, Erik Ramírez-Moctezuma ${ }^{b}$,
}

Liliana J. Sevilla Cervantes ${ }^{c}$, Teodoro Suarez-Diéguez ${ }^{d}$

\begin{abstract}
:
Nowadays, it has been of great interest to the scientific community to study the characterization of the bioactive components of Jamaica flower (Hibiscus sabdarifa L.). In this sense, Mexico has started the development of native Creole varieties of the country and there are no published studies of these varieties and the works reported are varieties of Asian and African origin. The objective of the work was to determine the content of bioactive compounds and antioxidant activity in vitro of the extracts obtained from the flower of Jamaica (Hibiscus sabdarifa) of Creole variety originated in the state of Michoacán, Mexico. The results obtained indicate that there were no statistically significant differences in the yield of the extracts under any of the experimental conditions in this study. However, the total phenolic content was found to be higher in the hydroalcoholic extract (80:20) at the two experimental temperatures $(1007.6 \pm 0.0 \mathrm{mg}$ EAG / 1 and $1009.3 \pm 0.0 \mathrm{mg}$ EAG / L). The aqueous extract $(100 \%)$ had a higher content of flavonoids $(23.5 \pm 0.0 \mathrm{mg}$ Eq of quercetin / $\mathrm{g})$, betanins $(177.00 \pm 0.0 \mathrm{mg}$ Eq of pigment / L) and betaxanthines $(131.7 \pm 0.0 \mathrm{mg} \mathrm{Eq}$ of pigment / L) compared to the other extracts. The anthocyanin content was higher in the hydroalcoholic extract at $25^{\circ} \mathrm{C}(1.5 \pm$ $0.0 \mathrm{mg} \mathrm{Eq}$ of cyanidine-3-glucoside / L). The antioxidant capacity measured by ABTS and DPPH resulted in a higher hydroalcoholic extract $(73.62 \pm 0.04$ and $220.09 \pm 0.05 \mu \mathrm{mol}$ Eq of Trolox / L). The chelating activity was higher in the $100 \%$ aqueous extracts $\left(52.05 \%\right.$ ) at a temperature of $50^{\circ} \mathrm{C}$. In conclusion, the $100 \%$ aqueous extract at a temperature of $50{ }^{\circ} \mathrm{C}$ obtained a higher content of flavonoids, betalains and betaxanthines and chelating activity compared to the rest of the extracts. The extract at an 80:20 ratio (water:ethanol), at a temperature of $25^{\circ} \mathrm{C}$ showed a greater amount of total phenolic and anthocyanin content, and these extracts showed a higher antioxidant activity compared to the rest of the extracts.
\end{abstract}

Keywords:

Hibiscus sabdariffa, extract, bioactive compounds, antioxidant activity in vitro.

Resumen:

Hoy en día, ha sido de gran el interés para la comunidad científica el estudiar la caracterización de los componentes bioactivos de flor de Jamaica (Hibiscus sabdarifa L.). En este sentido, México ha iniciado el desarrollo de variedades criollas nativas del país y no existen estudios publicados de estas variedades y los trabajos reportados son variedades de procedencia asiáticas y africanas. El objetivo del trabajo fue determinar el contenido de compuestos bioactivos y actividad antioxidante in vitro de los extractos obtenidos de flor de Jamaica (Hibiscus sabdarifa) de variedad criolla originaria del estado de Michoacán, México. Los resultados obtenidos indican que no hubo diferencias estadísticamente significativas en cuanto al rendimiento de los extractos bajo ninguna de las condiciones de experimentación en este estudio. Sin embargo, el contenido de fenoles totales resulto ser mayor en el extracto hidroalcoholico (80:20) en las dos temperaturas de experimentación (1007.6 $\pm 0.0 \mathrm{mg} \mathrm{EAG/1} \mathrm{y} \mathrm{1009.3 \pm 0.0} \mathrm{mg} \mathrm{EAG/L).} \mathrm{El} \mathrm{extracto}$ acuoso $(100 \%)$ presento un mayor contenido de flavonoides $(23.5 \pm 0.0 \mathrm{mg}$ Eq de quercetina/g), betaninas $(177.00 \pm 0.0 \mathrm{mg}$ Eq de pigmento/L) y betaxantinas $(131.7 \pm 0.0 \mathrm{mg} \mathrm{Eq}$ de pigmento/L) en comparación con los demás extractos. El contenido de antocianinas fue mayor en el extracto hidroalcohólico a $25{ }^{\circ} \mathrm{C}(1.5 \pm 0.0 \mathrm{mg} \mathrm{Eq}$ de cianidina-3-glucosido/L). La capacidad antioxidante medida por ABTS Y DPPH resulto fue mayor el extracto hidroalcohólico $(73.62 \pm 0.04$ y $220.09 \pm 0.05 \mu$ mol Eq de Trolox/L). La actividad quelante fue mayor en los extractos $100 \%$ acuosos $(52.05 \%)$ a una temperatura de $50{ }^{\circ} \mathrm{C}$. En conclusión, el extracto $100 \%$ acuoso a una temperatura de $50{ }^{\circ} \mathrm{C}$ obtuvo un contenido mayor de flavonoides, betalainas y betaxantinas y actividad quelante en comparación con el resto de los extractos El extracto a una relación 8020 (agua etanol), a una temperatura de $25{ }^{\circ} \mathrm{C}$

${ }^{a}$ Universidad Autónoma del Estado de Hidalgo, Instituto de Ciencias de la Salud ,Email: : envl_9206@hotmail.com

b Universidad Autónoma del Estado de Hidalgo, Instituto de Ciencias de la Salud, Email: erikram6@gamail.com

c Universidad Autónoma del Estado de Hidalgo, Instituto de Ciencias de la Salud, Email: : analili_23_@hotmail.com

dAutor de Correspondencia Universidad Autónoma del Estado de Hidalgo, Instituto de Ciencias de la Salud, Email: tsuarez@uaeh.edu.mx 
mostró mayor cantidad en contenido de fenoles totales y antocianinas, y estos extractos presentaron mayor actividad antioxidante en comparación con el resto de los extractos.

\section{Palabras Clave:}

Hibiscus sabdariffa, extracto, compuestos bioactivos, actividad antioxidante in vitro.

\section{Introducción}

Actualmente se ha hecho un énfasis en investigar a los fitoquímicos contenidos en los alimentos de origen vegetal, han mostrado actividad biológica al ser consumidos. Estos pueden generar un efecto benéfico sobre el metabolismo del ser humano y mejorar la condición de salud del individuo, a este efecto se le denomina actividad biológica. Los compuestos bioactivos son componentes de los alimentos que tienen una influencia en la actividad celular, en los mecanismos fisiológicos y con efectos benéficos para la salud (1). Las actividades biológicas que pueden presentan los compuestos bioactivos son antiinflamatorias, antioxidantes, quimiopreventivas y neuroprotectoras. entre otras. El consumo de alimentos vegetales se asocia a un riesgo menor de padecer enfermedades cardiovasculares, cáncer y diabetes (3).

Los polifenoles son fitoquímicos y pueden ser considerados compuestos bioactivos, es un grupo predominante y heterogéneo que se encuentran en frutos, hortalizas y leguminosas (2). Los polifenoles son un grupo de fitoquímicos que contienen anillos de fenol. Estos se dividen en flavonoides, ácidos fenólicos, estilbenos y lignanos. Los flavonoides incluyen a las flavonas, isoflavonas y antocianinas (4).

Hoy en día, se muestra un interés en estudiar la caracterización de los componentes bioactivos de la flor de Jamaica (Hibiscus sabdarifa L.), por el tipo de compuestos que contiene y las actividades biológicas que se han reportado, que pueden ejercer un efecto benéfico a la salud (antioxidantes, anticancerígenos, cardioprotectores y antihipertensivos) (3). Sin embargo, las variedades mexicanas han sido poco estudiadas.

El presente trabajo tuvo por objetivo determinar el contenido de compuestos bioactivos y actividad antioxidante in vitro de los extractos obtenidos de flor de Jamaica (Hibiscus sabdarifa) de variedad criolla originaria del estado de Michoacán, México.

\section{Materiales y métodos}

\subsection{Material biológico}

Se utilizó flor de Jamaica (Hibiscus sabdariffa) variedad criolla cultivada orgánicamente en Michoacán, México; adquirida de la comercializadora Ki-An S. A de C. V. Los reactivos utilizados fueron Folin de Ciocalteu, quercetina, ABTS, DPPH, trolox, cloruro férrico, ferrocina, obtenidos de Sigma-Aldrich, St. Louis, USA.

\subsection{Extracción}

La flor de Jamaica fue deshidratada por método convencional, posteriormente fue homogenizada la muestra hasta un tamaño de partícula de 200 micras y tamizado para obtener la homogenización de la muestra. El proceso de extracción consistió en realizar una maceración de la muestra por 24 horas considerando: a) temperatura de extracción $\left(25^{\circ} \mathrm{C}\right.$ y $\left.50{ }^{\circ} \mathrm{C}\right)$; b) mezclas de solventes agua 100\%, agua: etanol en una relación 80:20 y 50:50, ambos procedimientos se les adicionó 0.5 $\mathrm{ml}$ de ácido cítrico, para la estabilidad (5).

\subsection{Cuantificación de compuestos bioactivos}

Evaluación del contenido y actividad antioxidante de los compuestos bioactivos del extracto de flor de jamaica, fueron realizadas mediante técnicas espectrofotométricas con algunas modificaciones. 


\section{Hidalgo, Vol. 8, No. 15 (2019) 174-179}

\subsubsection{Fenoles totales}

El contenido de fenoles totales se realizó mediante la técnica reportada de Stintzing (6), se utilizó una solución de carbonato de sodio al $7.5 \%$ y Folin diluido $1: 10$. A una muestra de $100 \mu \mathrm{L}$ fue adicionado $500 \mu \mathrm{L}$ de Folin y $400 \mu \mathrm{L}$ de carbonato de sodio y agitados en el vórtex. Posteriormente se dejó reposar la mezcla durante 30 minutos. Finalmente fue medida la absorbancia de las muestras a $765 \mathrm{~nm}$ empleando un lector de microplacas (Power Wave XS [Bio Tek]. Software KC Junior, USA).

\subsubsection{Flavonoides}

El contendido de flavonoides totales fue determinado mediante la técnica reportada por Soares, Bassani, González Ortega y Petrovick (7). 400 mg de muestra fueron mezclados en un matraz con $1 \mathrm{~mL}$ de hexametilentetramina al 5\%, se adicionó $20 \mathrm{~mL}$ de acetona y $2 \mathrm{~mL}$ de ácido clorhídrico a la mezcla, fue calentada a reflujo en baño María por 30 minutos y posteriormente fue filtrada. El residuo fue lavado con 20 $\mathrm{mL}$ de acetona y aforado a $100 \mathrm{~mL}$ con acetona. Se tomaron $20 \mathrm{~mL}$ para extracción, mezcladas con $15 \mathrm{~mL}$ de acetato de etilo. De la mezcla anterior fueron tomados dos $10 \mathrm{~mL}$ de la solución y mezcladas con 2 $\mathrm{mL}$ de $\mathrm{AlCl}_{3}$ al $2 \%$ en etanol, la muestra fue aforada a 25 $\mathrm{mL}$ con una solución metanólica de ácido acético al $0.5 \%$. 30 minutos después fueron tomadas las lecturas espectrofotométricas a $420 \mathrm{~nm}$. El resultado fue expresado como miligramos de equivalentes de quercetina por gramo (mg EQ/g).

\subsubsection{Betalainas}

La cuantificación de betalainas se realizó mediante el método reportado por Castellar, Obón, Alacid y Fernández-López (8); Stintzing (6). $10 \mathrm{~mL}$ de muestra fueron centrifugadas (Hanna Instruents $\mathrm{pH} 210$, Romania) a $3500 \mathrm{rpm}$ durante $30 \mathrm{~min}$ a temperatura ambiente. Posteriormente fueron tomadas alícuotas de $200 \mu \mathrm{L}$ de cada muestra y colocadas en una microplaca y fue tomada la lectura de absorbancia a $480 \mathrm{~nm}$ para los pigmentos amarillos (betaxantinas) y a $535 \mathrm{~nm}$ para pigmentos rojos-purpura (betaninas).

Los resultados fueron expresados con la siguiente ecuación:

mg Eq de pigmento/L = $(A \times F D \times P M \times 1000 / \epsilon \times I)$

Dónde:

A: absorbancia a 535 o $480 \mathrm{~nm}$

FD: factor de dilución

PM: peso molecular

$€$ : coeficiente de extinción molecular

I: volumen de cada celda $\left(1 \mathrm{~cm}^{3}\right)$

Los coeficientes de extinción molar son:

Betacianinas: $\Theta=60000 \mathrm{~L}(\mathrm{~mol} \mathrm{~cm}), P M=550 \mathrm{~g} / \mathrm{mol}$

Betaxantinas $\Theta=48000 \mathrm{~L}(\mathrm{~mol} \mathrm{~cm}) \mathrm{PM}=308 \mathrm{~g} / \mathrm{mol}$

\subsubsection{Antocianinas}

El contenido de antocianinas fue determinado de acuerdo a la técnica reportada por Prior (9), con algunas modificaciones, considerando estimar la cuantificación mediante el método de $\mathrm{pH}$ diferencial. Fueron tomadas las lecturas de absorbancia en un espectrofotómetro a $510 \mathrm{~nm}$ y $700 \mathrm{~nm}$, ambas a pH de 1,0 y 4,5, considerando un coeficiente de extinción molar para la cianidina-3-glucosido de 29600. Los resultados fueron expresados como miligramos de equivalente de cianidina-3-glucosido por $100 \mathrm{mg}$ de peso fresco.

\subsection{Determinación de actividad antioxidante}

\subsubsection{Actividad antioxidante por $\mathrm{ABTS}^{\bullet+}$}

La actividad antioxidante por $\mathbf{A B T S}^{\bullet+}$ fue determinada de acuerdo al método descrito por Kuskoski, Asuero, García-Parilla, Troncoso y Fett (10), con algunas modificaciones.

La determinación fue realizada mediante la formación del radical $A B T S$, el cual se obtiene tras la reacción de ABTS (7 mM) con persulfato potásico (2.45 mM), incubados a temperatura ambiente $\left( \pm 25^{\circ} \mathrm{C}\right)$ y en 


\section{Hidalgo, Vol. 8, No. 15 (2019) 174-179}

condición de oscuridad por un periodo de 16 horas. Una vez formado el radical ABTS la muestra fue diluida hasta obtener un valor de absorbancia entre $0.70( \pm 0.1)$ a 754 $\mathrm{nm}$. A $20 \mu \mathrm{L}$ de muestra fueron agregados $980 \mu \mathrm{L}$ de solución de ABTS, se agitó en el vórtex y se dejó reposar a temperatura ambiente por 7 minutos. Finalmente fue tomada la absorbancia a $754 \mathrm{~nm}$ empleando un lector de microplacas (Power Wave XS [Bio Tek]. Software KC Junior, USA).

\subsubsection{Actividad antioxidante por DPPH $^{\bullet}$}

La actividad de DPPH $^{\bullet}$ fue determinada de acuerdo al procedimiento reportado por Morales y Jiménez-Pérez (11) con algunas modificaciones. A $100 \mu \mathrm{L}$ de muestra fue mezclada con $500 \mu \mathrm{L}$ de solución $\mathrm{DPPH}$, fue homogenizada por agitación en el vórtex y se dejó reposar a temperatura ambiente por una hora. Posteriormente se determinó la absorbancia a $520 \mathrm{~nm}$, empleando lector de microplacas (Power Wave XS [Bio Tek]. Software KC Junior, USA). Fue utilizado Trolox como antioxidante de referencia $(3.75 \mathrm{mg}$ del reactivo diluidos en $50 \mathrm{ml}$ de etanol de grado analítico). Los resultados fueron expresados en $\mu \mathrm{mol}$ de equivalentes de Trolox por $100 \mathrm{~g}$ de muestra.

\subsubsection{Actividad quelante}

La actividad quelante fue determinada mediante el método reportado por Xie, Huang, Xu y Jin (12) con algunas modificaciones. Se diluyeron $50 \mathrm{~mL}$ de solución de ferrocina en $950 \mathrm{~mL}$ de agua destilada. A $100 \mu \mathrm{L}$ de muestra fueron adicionados $50 \mu \mathrm{L}$ de solución de cloruro férrico más $450 \mu \mathrm{L}$ de metanol, fueron mezcladas con agitación y se dejaron reposar 5 minutos. Posteriormente fueron agregados $400 \mu \mathrm{L}$ de ferrocina, y mezcladas con agitación, se dejaron reposar otros 10 minutos. Fue generado un blanco con el mismo procedimiento con agua y EDTA. El porcentaje de actividad quelante fue calculado de la siguiente forma:
$\%$ de Actividad quelante $=\left(\left(A_{0}-A_{1}\right) / A_{0}\right) \times 100$

Dónde:

$A_{0}=$ Absorbancia de la muestra control

$A_{1}=$ Absorbancia de la muestra

\subsection{Análisis estadístico}

Las determinaciones por triplicado fueron expresadas como la media de los ensayos +/- su desviación estándar. El análisis estadístico se realizará mediante un análisis de varianza de una vía (ANOVA) y la significancia estadística fue considerada con $\mathrm{p}<0.05$. Fue realizado el análisis con el programa SPSS versión 24 ,

\subsection{Resultados y discusión}

En la Tabla 1 se muestran los diferentes extractos obtenidos de la flor de Jamaica bajo las condiciones de experimentación de este estudio.

El rendimiento obtenido de los extractos no presenta diferencias estadísticamente significativas $(p<0.05)$ para ninguna de las condiciones de extracción. Esto se debe a que las desviaciones estándar fueron muy elevadas, lo cual puede deberse a un sesgo de error en el proceso de elaboración.

Tabla 1: Rendimiento de Extracción.

\begin{tabular}{ccccc}
\hline Extracto & Agua \% & Etanol \% & Temperatura ${ }^{\circ} \mathbf{C}$ & Rendimiento \% \\
\hline $\mathbf{1 . 1}$ & 80 & 20 & 25 & $37.12 \pm 4.14^{\mathrm{a}}$ \\
$\mathbf{1 . 2}$ & 80 & 20 & 50 & $45.54 \pm 4.36^{\mathrm{a}}$ \\
$\mathbf{2 . 1}$ & 50 & 50 & 25 & $42.30 \pm 7.07^{\mathrm{a}}$ \\
$\mathbf{2 . 2}$ & 50 & 50 & 50 & $47.56 \pm 7.32^{\mathrm{a}}$ \\
$\mathbf{3 . 1}$ & 100 & 0 & 25 & $41.81 \pm 13.19^{\mathrm{a}}$ \\
$\mathbf{3 . 2}$ & 100 & 0 & 50 & $44.75 \pm 10.01^{\mathrm{a}}$ \\
\hline
\end{tabular}

Los valores son las medias \pm SD. $(n=3)$. Diferentes letras superíndice indican diferencias significativas $(p<0.05)$.

En la cuantificación de los diferentes compuestos bioactivos, se observa un mayor contenido de fenoles (1007.0 $\pm 0.0 \mathrm{mg} E A G / L)$ y antocianinas $(1.5 \pm 0.0)$ para el extracto hidroalcohólico 80:20, a una temperatura de 25 ${ }^{\circ} \mathrm{C}$. Sin embargo, en el extracto acuoso (100\% agua) a temperatura de $50{ }^{\circ} \mathrm{C}$ mostró tener un contenido mayor de flavonoides $(23.5 \pm 0.0 \mathrm{mg} \mathrm{EQ} / \mathrm{mg})$, betaninas $(177.0 \pm 0.0 \mathrm{mg} \mathrm{Eq}$ de pigmento/L) y betaxantinas $(131.7 \pm 0.0 \mathrm{mg} \mathrm{Eq}$ de pigmento/L) (Tabla 2). 
Tabla 2: Cuantificación de compuestos bioactivos en los extractos.

\begin{tabular}{|c|c|c|c|c|c|}
\hline \multirow[b]{2}{*}{ 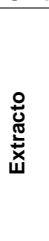 } & \multirow{2}{*}{ 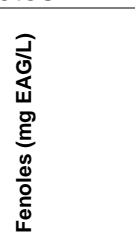 } & \multirow{2}{*}{ 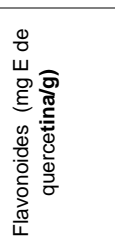 } & \multicolumn{2}{|c|}{$\begin{array}{c}\text { Betalainas } \\
\text { ( } \mathrm{mg} \mathrm{E} \text { de pigmento/L) }\end{array}$} & \multirow{2}{*}{ 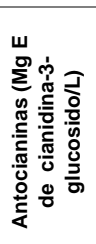 } \\
\hline & & & 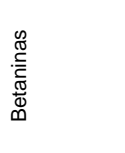 & 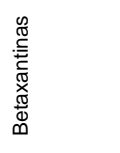 & \\
\hline 1.1 & a & D & . & D & $\bar{a}$ \\
\hline 1.2 & $1007.6 \pm 0.0$ & $16.6 \pm 0.0_{D}$ & $54.9 \pm 0.00{ }_{\mathrm{c}}$ & $35.6 \pm 0.00$ & $1.5 \pm 0.0_{\mathrm{c}}$ \\
\hline 2.1 & $1009.3 \pm 0.0$ & $17.5 \pm 0.0$ & $46.7 \pm 0.00$ & $33.6 \pm 0.0$ & $0.8 \pm 0.0_{b}$ \\
\hline 2.2 & $505.1 \pm 0.0_{\mathrm{e}}$ & $8.2 \pm 0.0$ & $15.5 \pm 0.0$ & $9.8 \pm 0.0$ & $0.9 \pm 0.0 \mathrm{c}$ \\
\hline & $472.6 \pm 0.0$ & $10.2 \pm 0.0$ & $20.3 \pm 0.0$ & $14.6 \pm 0.0$ & $0.5 \pm 0.0$ \\
\hline 3.1 & c & c & a & c & c \\
\hline 3.2 & $769.3 \pm 0.1_{\mathrm{D}}$ & $12.5 \pm 0.0$ & $26.9 \pm 0.0$ & $19.1 \pm 0.0$ & $0.6 \pm 0.0$ \\
\hline & $786.0 \pm 0.0$ & $23.5 \pm 0.0$ & $177.0 \pm 0.0$ & $131.7 \pm 0.0$ & $0.8 \pm 0.0$ \\
\hline
\end{tabular}

Los valores son las medias \pm SD. $(n=3)$. Diferentes letras superíndice indican diferencias significativas $(p<0.05)$.

Esto se debe a que se le adicionó citrato al $0.5 \mathrm{M}$ con la finalidad de estabilizar los compuestos y generar un $\mathrm{pH}$ ácido, ya que Morales-Luna (13) menciona que a un $\mathrm{pH}$ ácido se presenta una mayor cantidad de ácidos orgánicos en extractos elaborados con solventes acuosos.

En un estudio realizado por Higginbotham et al. (14) reportaron que el contenido de fenoles totales en extracto acuoso, fue de $1600 \mathrm{mg}$ EAG/L para la variedad alma blanca. Además, reportaron que las diferencias en el contenido de compuestos bioactivos de flor de Jamaica puede atribuirse a las condiciones de extracción.

El estudio de Christian y Jackson (15) reportando que contenido de fenoles totales de extracto etanólicos fue de $2312 \mathrm{mg}$ EAG/L, presentando un contenido mayor a este estudio.

Otro estudio realizado por Oyindamola (16) obtuvieron extractos acuosos y metanólicos de variedades rojas y blancas de $H$. sabdariffa. Este grupo reporto que los extractos acuosos de ambas variedades muestran tener un mayor contenido de ácidos orgánicos comparados con el extracto metanólico, mientras que los fenoles, antocianinas y flavonoides fueron más altos en el extracto metanólico.

La máxima actividad antioxidante por los métodos ABTS Y DPPH se obtuvo en el extracto hidoalcohólico con
$73.62 \pm 0.04$ y $220.09 \pm 0.05$ micromol equivalente de Trolox por litro (Figura 1 y figura 2).

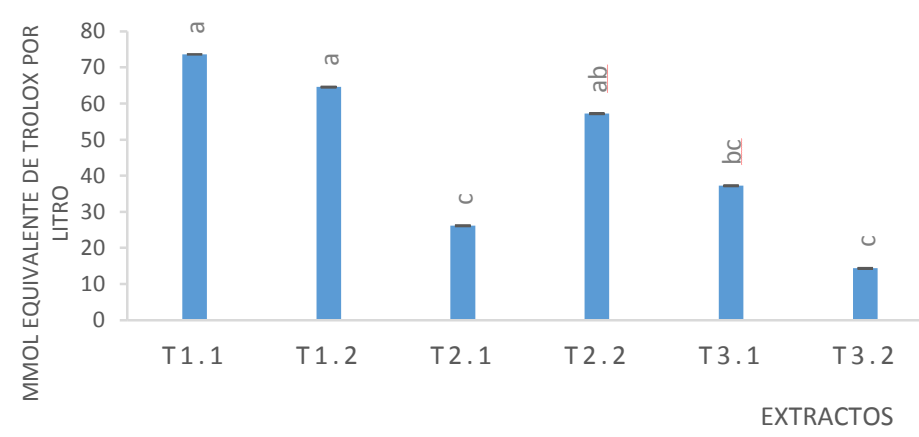

Figura 1: Actividad antioxidante in vitro por el método ABTS Los valores son las medias \pm SD. $(n=3)$. Diferentes letras superíndice indican diferencias significativas $(p<0.05)$.

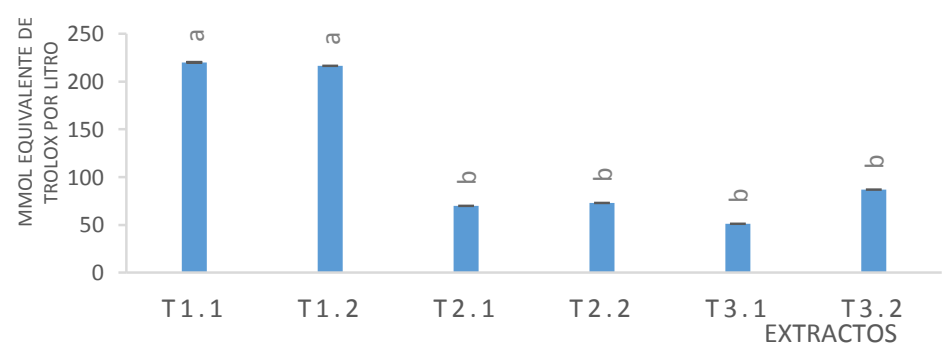

Figura 2: Actividad antioxidante in vitro por el método DPPH Los valores son las medias $\pm \operatorname{SD}$. $(n=3)$. Diferentes letras superíndice indican diferencias significativas $(p<0.05)$.

Estos resultados se atribuyen a las condiciones de extracción ya que en extractos hidroalcohólicos se presenta una mayor actividad antioxidante (17).

En cuanto a actividad quelante el porcentaje se observa en la figura 3, donde el más alto fue el extracto acuoso con un $52.05 \%$.

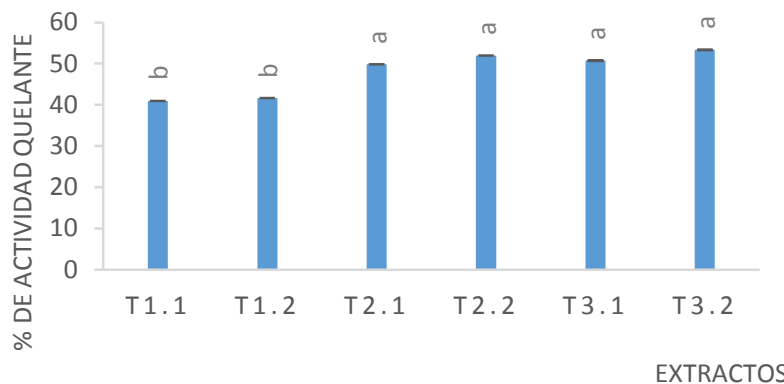

Figura 3: Porcentaje de actividad Quelante

Los valores son las medias $\pm \operatorname{SD}$. $(n=3)$. Diferentes letras superíndice indican diferencias significativas $(p<0.05)$. 
Por otro lado, en la investigación realizada por VargasLeon et al (2018) obtuvieron resultados similares a este estudio en el contenido de flavonoides, antocianinas y actividad antioxidante por ABTS y DPPH reportando que las concentraciones de flavonoides y antocianinas son más altas en extractos acuosos, asociando sus resultados obtenidos a las condiciones de extracción, estas diferencias en el nivel de actividad antioxidante en los diferentes extractos, puede ser debido a la oxidación de compuestos fenólicos, que pueden ser destruidas como es el caso de las catequinas.

\section{Conclusión}

El extracto de $100 \%$ agua a una temperatura de $50{ }^{\circ} \mathrm{C}$ obtuvo un contenido mayor de flavonoides, betalainas y betaxantinas, y actividad quelante en comparación con el resto de los extractos. El extracto elaborado a una relación 80:20 (agua: etanol), a una temperatura de 25 ${ }^{\circ} \mathrm{C}$ mostro mayor cantidad en contenido de fenoles totales y antocianinas, y estos extractos presentaron mayor actividad antioxidante en comparación con el resto de los extractos.

\section{Referencias}

[1] Hanhineva K, Törrönen R, Bondia-pons I, Pekkinen J, Kim Y, Keogh JB, et al. Polyphenols and Glycemic Control. 2016; $2471: 1365-1402$.

[2] Zatalia SR, Sanusi H. The role of antioxidants in the pathophysiology, complications, and management of diabetes mellitus. Acta Med Indones. 2013;45:141-148.

[3] Howes MR, Simmonds MSJ. The role of phytochemicals as micronutrients in health and disease. 2014;17:558-566.

[4] Kim Y, Keogh JB, Clifton PM. Polyphenols and Glycemic Control. 2016;2471: 1-27.

[5] Dewanjee, S., Das, A. K., Sahu, R. y Gangopadhyay, M. Antidiabetic activity of Diospyros peregrina fruit: Effect on hyperglycemia, hyperlipidemia and augmented oxidative stress in experimental type 2 diabetes. Food and Chemical Toxicology. 2009; 47: 2679-2685.

[6] Stintzing, F. C., Herbach, K. M., Mosshammer, M. R., Carle, R., Yi W, Sellappan S, Felker P. Color, betalain pattern, and antioxidant properties of cactus pear (Opuntia spp.) clones. Journal of Agricultural and Food Chemistry. 2005; 53: 442451

[7] Soares LA, Bassani V L, González Ortega G, y Petrovick PR. Total Flavonoid Determination for the Quality Control of
Aqueous Extractives from Phyllanthus niruri L. Acta Farmaceutica Bonaerense. 2003; 22: 203-207.

[8] Castellar R., Obón J M, Alacid, M y Fernández-López JA. Color properties and stability of betacyanins from Opuntia fruits. Journal of Agricultural and Food Chemistry. 2003;51: 2772-2776.

[9] Prior R. L, Cao G, Martin A, Sofic E, Mc Ewen J, O’Brien C, Mainland CM. Antioxidant Capacity As Influenced by Total Phenolic and Anthocyanin Content, Maturity, and Variety of Vaccinium Species. Journal of Agricultural and Food Chemistry. 1998; 46: 2686-2693

[10] Kuskoski EM, Asuero AG, García-Parilla MC, Troncoso A., y Fett R.. Actividad antioxidante de pigmentos antociánicos. Ciência e Tecnologia de Alimentos. 2004; 24: 691-693.

[11] Morales FJ, y Jiménez-Pérez S. Free radical scavenging capacity of Maillard reaction products as related to colour and -uorescence. Food Chemistry. 2001; 72: 119-125.

[12] Xie Z, Huang J, Xu X, y Jin Z. Antioxidant activity of peptides isolated from alfalfa leaf protein hydrolysate. Food Chemistry. 2008; 111: 370-376.

[13] Morales-Luna E, Perez-Ramírez IF, Salgado LM, Casaño-Tostado E, Gómez-Aldapa CA y Reinoso-Camacho R. The main beneficiasl effect of roselle (Hibiscus sabdariffa) on obesity is not only related to its anthocyanin content. J. sci food agric. 2018; 99: 596-605.

[14] Higginbotham K.L, Burris K.P, Zivanovic S, Davidson P.M., Stewart CN. Aqueous extracts of Hibiscus sabdariffa calyces as an antimicrobial rinse on hot dogs against Listeria monocytogenes and methicillin-resistant Staphylococcus aureus. Food Control. 2014; 40: 274-277

[15] Christian KR, Nair MG, Jackson JC. Antioxidant and cyclooxygenase inhibitory activity of sorrel (Hibiscus sabdariffa). J. Food Comp. 2006; 19: 778-783.

[16] Oyindamola-Vivian O, Seul-Gi L, Ju-Ock N. Beneficial effects of natural bioactive compounds from Hibiscus sabdariffa L. on obesity. Molecules. 2019; 24: 1-14.

[17] Mercado-Mercado G, Blancas-Benitez FJ, Velderrain-Rodriguez G. Bioaccessibillity of polyphenols released and associated to dietary fibre in calyces and decoction residues of Roselle (Hibiscus sabdariffa). Journal of functional foods. 2015; 18: 171-181.

[18] Vargas-León EA, Díaz-Batalla L, González-Cruz L, Bernardino-Nicanor A, Castro-Rosas J, Reynoso-Camacho R, et al. Effects of acid hydrolysis on the free radical scavenging capacity and inhibitory activity of the angiotensin converting enzyme of phenolic compounds of two varieties of jamaica (Hibiscus sabdariffa). Ind Crops Prod. 2018;116: 201-8. 